\section{Finasteride improves capacity of the PSA test to detect prostate cancer}

The results of the Prostate Cancer Prevention Trial (PCPT) revealed that patients receiving finasteride had a lower risk of developing prostate cancer, but a higher risk of high-grade cancer, compared with those on placebo. Thompson et al. investigated whether treatment with finasteride could have affected the diagnostic performance of the PSA test.

This study included all men in the finasteride and placebo groups who underwent prostate biopsy and PSA testing during the 7-year PCPT. The effects of finasteride on the sensitivity of and the area under the receiver-operatingcharacteristic curve (AUC) of PSA tests for detection of prostate cancer by biopsy were investigated. Sensitivity was defined as the proportion of patients with PSA levels exceeding a series of cutoff values.

The finasteride group included 4,579 men, 695 of whom were diagnosed with prostate cancer. Of these, $38.5 \%$ had a Gleason score of $\geq 7$. The placebo group consisted of 5,112 men, 1,111 of whom were diagnosed with prostate cancer. Of these, $21.8 \%$ had a Gleason score of $\geq 7$. For all outcomes, the AUC was greater for the finasteride group than for the placebo group. At all PSA cutoffs, the sensitivity of PSA was greater in the finasteride group than in the placebo group.

Finasteride seemed to enhance the sensitivity and AUC of PSA testing for detecting all grades of prostate cancer. The higher risk of high-grade prostate cancer in the PCPT was partly a result of increased sensitivity of PSA, rather than a result of the induction of disease by finasteride.

Original article Thompson IM et al. (2006) Effect of finasteride on the sensitivity of PSA for detecting prostate cancer. J Natl Cancer Inst 98: 1128-1133

\section{Children with a single, normal kidney can participate in contact/collision sports}

Consensus is lacking among medical professionals as to whether children and adolescents with a single, normal kidney should participate in contact/collision sports; faced with confusing guidance, many advise against participation. Contrary to this opinion, Grinsell and colleagues at the University of Virginia declare that precluding these patients from such activities is unwarranted.

The investigators analyzed data from a survey of members of the American Society of Pediatric Nephrology, and information retrieved from searches of literature databases on sports-related kidney, brain, spinal cord and cardiac injury. In total, $62 \%$ of respondents barred participation in contact/collision sports, the majority citing risk of renal injury and consequences of subsequent loss of function as primary reasons. According to the medical and sports data, cycling and skiing were the most dangerous sports in terms of damage to the kidney; American football, regarded as the most dangerous sport by $86 \%$ of the survey respondents who banned sports participation, caused only about a third the number of injuries. Many more catastrophic injuries of the spinal cord, brain, and heart were recorded than of the kidney. For all sports, the incidence of catastrophic sportsrelated kidney injury was only 0.4 per million children per year.

Grinsell et al. believe that the benefits to be gained from sports participation outweigh the small risk of renal injury, and recommend that current practices and guidelines are reviewed and clarified.

Original article Grinsell MM et al. (2006) Single kidney and sports participation: perception versus reality. Pediatrics 118: 1019-1027 